\title{
Colossal variations in the thermopower and $n-p$ conductivity switching in topological tellurides under pressure
}

Cite as: J. Appl. Phys. 128, 245902 (2020); doi: 10.1063/5.0031818

Submitted: 4 October 2020 - Accepted: 3 December 2020 .

Published Online: 22 December 2020

Natalia V. Morozova, ${ }^{1, a)}$ (D) Igor V. Korobeinikov, ${ }^{7}$ (D) Kirill V. Kurochka, ${ }^{1,2}$ (D) and Sergey V. Ovsyannikov ${ }^{3,4, a) ~(D) ~}$

\begin{abstract}
AFFILIATIONS
${ }^{1}$ M. N. Miheev Institute of Metal Physics of Ural Branch of Russian Academy of Sciences, 18 S. Kovalevskaya Str., Yekaterinburg 620137, Russia

${ }^{2}$ Institute of Natural Sciences and Mathematics, Ural Federal University, 19 Mira St., Yekaterinburg 620002, Russia

${ }^{3}$ Bayerisches Geoinstitut, Universität Bayreuth, Universitätsstrasse 30, D-95447 Bayreuth, Germany

${ }^{4}$ Institute for Solid State Chemistry of Ural Branch of Russian Academy of Sciences, 91 Pervomayskaya St., Yekaterinburg 620219, Russia
\end{abstract}

Note: This paper is part of the Special Topic on Topological Materials and Devices.

${ }^{a)}$ Authors to whom correspondence should be addressed: morozova@imp.uran.ru; natasha_nt88@mail.ru; and sergey. ovsyannikov@uni-bayreuth.de

\begin{abstract}
Under applied high pressure, the electronic, optical, structural, and other properties of narrow-bandgap telluride semiconductors are subjected to dramatic changes. They can include, for instance, structural and electronic topological transitions. In this work, we investigated the electronic properties of single crystals of three families of tellurides, namely, $\mathrm{HgTe}, \mathrm{PbTe}$, and $\mathrm{Bi}_{2} \mathrm{Te}_{3}$ by measurements of the thermoelectric power (the Seebeck coefficient) and electrical resistance under high pressure up to $10 \mathrm{GPa}$. The applied pressure led to spectacular variations in the electronic transport of all three tellurides. We addressed these effects to electronic topological transitions that could be driven by significant narrowing of the bandgaps in the normal-pressure phases of these compounds. In particular, at about $1 \mathrm{GPa}$, we observed an $n-p$ switching in the conductivity of $\mathrm{HgTe}$, which was well reproducible under multiple pressure cycling. In contrast, in $\mathrm{PbTe}$, we found that an electronic topological transition irreversibly turns the conductivity from $p$ - to $n$-type. An electronic topological Lifshitz transition in $p$-type $\mathrm{Bi}_{2} \mathrm{Te}_{3}$ crystals with a low carrier concentration enhanced the $n$-type conductivity in a narrow pressure region about $2-3 \mathrm{GPa}$ and resulted in a double $p-n-p$ conductivity inversion. An irreversible $p-n$ conductivity switching in $p$-type $\mathrm{Bi}_{2} \mathrm{Te}_{3}$ happened already on decompression from a high-pressure phase from about $8 \mathrm{GPa}$. The stress-controlled $p-n$ inversions of the electrical conductivity in these industrially important telluride materials can potentially find emergent applications in micro- and nanoelectronics.
\end{abstract}

Published under license by AIP Publishing. https://doi.org/10.1063/5.0031818

\section{INTRODUCTION}

Controlling the electrical currents, which depend on the type and magnitude of electrical conductivity and the thermopower of materials, is crucially important for many semiconductor appliances. ${ }^{1}$ Therefore, different methods, which allow effectively control the electrical conductivity magnitude and its type ( $p$ - or $n-$ ) in various industrially relevant semiconductors, would open up prospects to novel applications. For instance, a possibility of elegant and reversible switching, the conductivity type in individual microor nanoscopic semiconductor elements will allow one to create in the future a new generation of $p-n$ switches, $p-n$ diode elements, $n-p-n(p-n-p)$ transistors, memory appliances, and various multilayer heterostructures with alternating types of electrical conductivity. ${ }^{2-7}$

The most common methods that are employed to vary the conductivity type are doping, ${ }^{8,9}$ implantation or irradiation, ${ }^{10,11}$ and stabilization of another structural phases with different 
electronic properties, e.g., in strained thin films. ${ }^{12,13}$ Conductivity type can also be inverted upon structural phase transitions, for example, under variation either in pressure $e^{5,7,14}$ or in temperature. ${ }^{15-20}$ Note that doping and implantation result in irreversible changes in electronic transport properties, whereas variations linked to pressure- or temperature-driven structural phase transitions are often reversible. However, in the latter case, a multiple cycling across phase transitions can severely damage specimens. Therefore, a search for new alternatives that could allow reliable and more elegant inversions of electronic properties remains a topical task.

It was established that band structure topology can have a noticeable effect on electronic properties of materials. ${ }^{21-31}$ Different methods like the above-discussed doping ${ }^{21,24,32-37}$ or pressure/ stress application ${ }^{25-31,38-43}$ can lead to significant changes in topology. Generally, electronic topological transitions can lead to different sorts of band structure variations. Transitions that are associated with changes in the Fermi surface topology are named as "Lifshitz transitions," and they were originally predicted in metals under compression. ${ }^{44}$ Under moderate high applied pressure, they were observed, for example, in $\mathrm{Bi}_{2} \mathrm{Te}_{3}{ }^{45}$ and SnSe. ${ }^{29}$ There are more spectacular electronic transitions that are linked to transformations from trivial to the non-trivial state of band structure topology. Such a transition was predicted, for instance, in $\mathrm{PbTe}$ under an applied pressure of a few $\mathrm{GPa}$ in its $\mathrm{NaCl}$ phase. ${ }^{46}$ The latter transition was also observed in $\mathrm{Bi}_{2} \mathrm{Te}_{3}$ and $\mathrm{Bi}_{2} \mathrm{Se}_{3}$ at ambient pressure under the appropriate level of $p$-doping. ${ }^{33,34}$ Hence, a controlled variation in the band structure topology is an effective method to tune the electronic properties.

Applied high pressure is capable to affect dramatically the electronic band structure of semiconductors and semimetals. ${ }^{25,27,28,30,47-49}$ For instance, at normal conditions, rock salt-structured $\mathrm{PbTe}$ has a direct bandgap of about $E_{g}=0.3 \mathrm{eV},{ }^{47-54}$ and its pressure coefficient $\left[\mathrm{d} E_{g} / \mathrm{d} P \approx-(70 \div 90) \mathrm{meV} / \mathrm{GPa}\right]^{47-54}$ suggests that this gap should be closed at about $3-4 \mathrm{GPa} .{ }^{47,55}$ However, a structural phase transition to the Pnma phase ${ }^{56-59}$ concurrent with a bandgap opening happens in PbTe only about 5.5$7 \mathrm{GPa} .{ }^{47,60,61}$ Thus, in the pressure range of about 3-6 GPa rock salt-structured $\mathrm{PbTe}$ should be in a highly unconventional nearly gapless state. It was found experimentally that the thermoelectric properties of $\mathrm{PbTe}$ are greatly enhanced in this 3-6 GPa range. ${ }^{62}$ As mentioned above, theoretical studies predicted for $\mathrm{PbTe}$ a transition from a trivial insulator to a topological one at $\sim 3.4-$ $4.8 \mathrm{GPa}^{46,49,63,64}$ The example of PbTe clearly demonstrates a possibility of profound alterations in the band structure topology, when applied high pressure progressively narrows a semiconductor bandgap. In its turn, it should lead to significant variations in electronic transport and other properties. Potentially, these effects, which are not linked to crystal-structure reconstructions, can be of significant technological interest for the semiconductor industry. However, because in situ high-pressure studies strongly restrict the set of experimental techniques feasible for investigation, the pressure-driven topological transitions and related changes in electronic properties remain poorly studied to date.

In this work, we investigated the effect of applied high pressure on the thermoelectric power and electrical resistance of three telluride families, including $\mathrm{HgTe}$ and its solid $\mathrm{HgTe}_{1-x} \mathrm{~S}_{x}$ solutions, PbTe crystals, and $\mathrm{Bi}_{2} \mathrm{Te}_{3}$ crystals, in which, the electronic topological transitions were expected. We found that the applied pressure can invert the type of the electrical conductivity in all these tellurides and proposed several simple examples of how these effects could be implemented.

\section{DETAILS OF EXPERIMENT}

For this study, we used single crystals of the tellurides from different suppliers. The solid solutions of $\mathrm{HgTe}_{1-x} \mathrm{~S}_{x}$ crystals with $x=0,0.05,0.10$, and 0.15 were synthesized in the quartz ampoules by melting together the highly pure (99.9999\%) components of $\mathrm{Hg}$, $\mathrm{Te}$, and S. The sample compositions were determined in a microprobe analysis using a Superprobe-JCXA-733. One crystal of $\mathrm{Bi}_{2} \mathrm{Te}_{3}$ was grown by the vertical Bridgman method. The crystals of PbTe were grown by the Czochralski technique.

The measurements of the thermoelectric power (the Seebeck coefficient) and the electrical resistance were carried out at room temperature in an anvil-type high-pressure cell of a toroidal type (both anvils have the semispherical cavities) under a continuous change in the applied pressure value up to $10 \mathrm{GPa} \cdot{ }^{65,66}$ A culet size of the anvils amounted to about $1 \mathrm{~mm}$. This cell allowed to investigate bulk samples with typical sizes of about $150 \times 150 \times 150 \mu \mathrm{m}^{3}$. ${ }^{66}$ A toroidal-shaped container that was made of limestone mineral served both as a gasket and as a pressure-transmitting medium. ${ }^{65,66}$ The high-pressure cell was loaded inside an automated mini-press setup. ${ }^{3,67}$ This setup allowed smoothly to increase and decrease an applied force and simultaneously to register automatically all relevant output signals. ${ }^{3,67}$ The force applied to the high-pressure cell was automatically measured by a digital dynamometer with the resistive-strain sensors. For each sample, the thermopower and the electrical resistance measurements were performed for several pressurization and decompression cycles. ${ }^{68}$ The electrical resistance was measured by a quasi-four-probe method (two bifurcated probes). ${ }^{4}$ In the thermopower measurements, a temperature difference $(\Delta T)$ across a sample was generated by heating of an upper anvil. Other details can be found in previous papers. ${ }^{65,66}$

\section{RESULTS}

\section{A. HgTe and $\mathrm{HgTe}_{1-\mathrm{x}} \mathrm{S}_{\mathrm{x}}$}

At normal conditions, mercury telluride, $\mathrm{HgTe}$ is a gapless semiconductor with an inverted band structure. ${ }^{69-75} \mathrm{HgTe}$ crystallizes in a zinc-blende (sphalerite)-type structure [Fig. 1(a)], which is transformed under applied high pressure, first, to a cinnabar-type structure at $1-3 \mathrm{GPa}$ [Fig. 1(b) $]^{76-94}$ and then, to a rock salt (NaCl-type) phase above 7-10 GPa [Fig. 1(c)]. ${ }^{82-85,87,88,95}$ Note that the cinnabar phase was predicted to be a good thermoelectric. ${ }^{96,97}$ The zinc-blende $\rightarrow$ cinnabar phase transition in HgTe was established to result in a bandgap opening. ${ }^{98-100}$ Binary HgTe was the focus of many experimental high-pressure studies, which investigated its structural, optical, and electronic transport properties, ${ }^{69,76,77,80,82-84,87,88,95,98-106}$ whereas its solid solutions, like $\mathrm{HgTe}_{1-x} \mathrm{~S}_{x}$, were considered in a few works only. ${ }^{89-91,105,107-109}$ It was found that a certain critical concentration of sulfur of about $x_{c} \sim 0.15-0.2$ turns $\mathrm{HgTe}_{1-x} \mathrm{~S}_{x}$ from a gapless semiconductor to a semimetal. ${ }^{89,107} \mathrm{HgTe}$ was reported to turn to a three-dimensional 
(a) Sphalerite-type

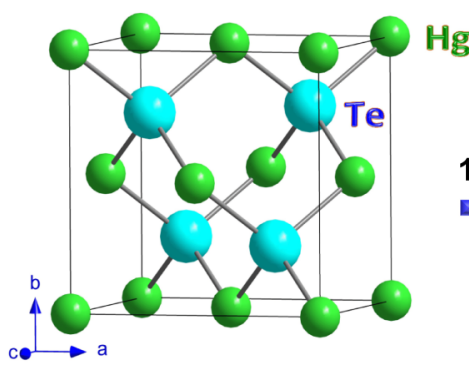

(d)

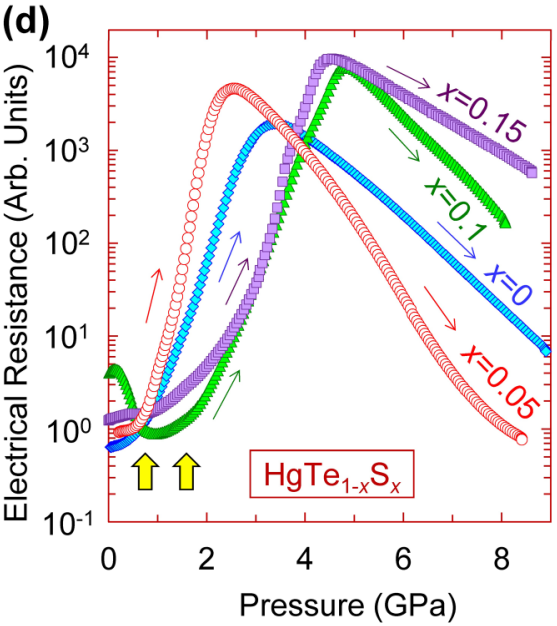

(b) Cinnabar-type

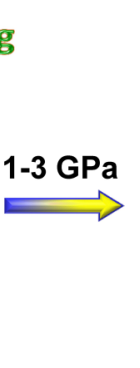

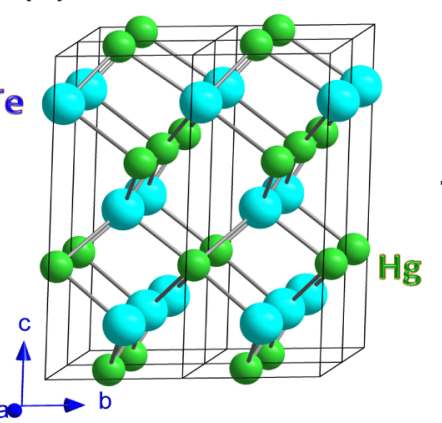

(e)

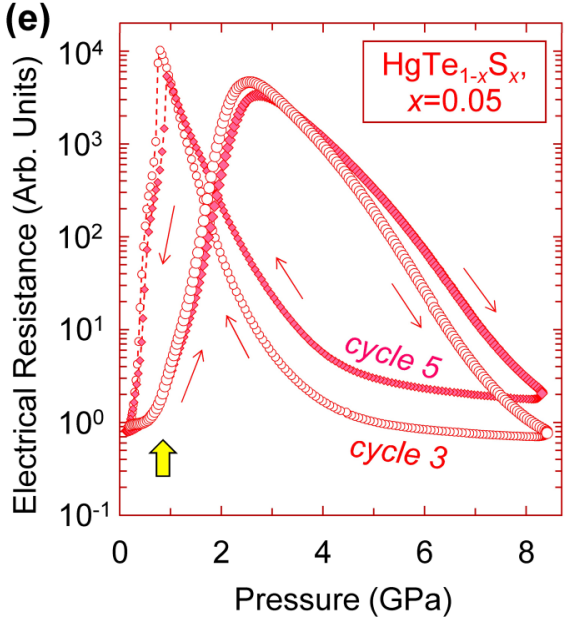

(c) Rock-salt-type

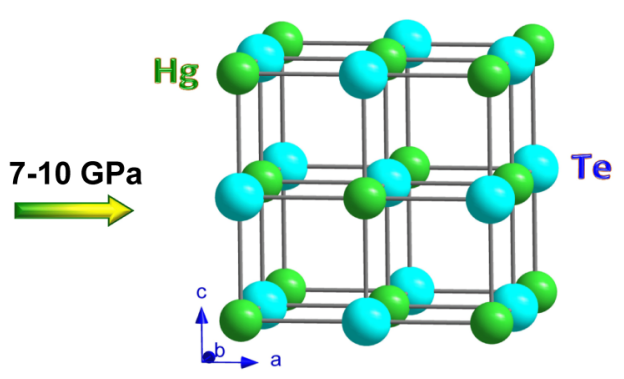

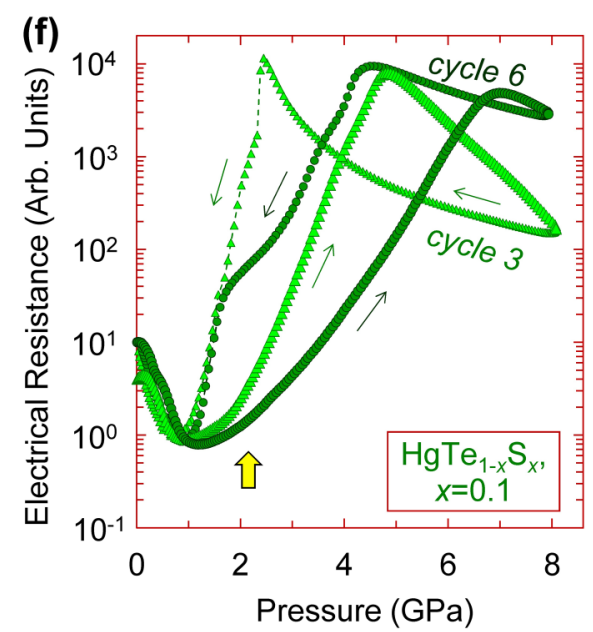

FIG. 1. (a)-(c) Room-temperature crystal structures of HgTe at different pressures: (a) sphalerite- or zinc-blende type, (b) cinnabar-type, and (c) rock salt-type (NaCl). (d) Pressure dependencies of the electrical resistance of $\mathrm{HgTe}_{1-x} \mathrm{~S}_{x}(x=0,0.05,0.1$, and 0.15$)$ single crystals across the sphalerite $\rightarrow$ cinnabar phase transition (pointed by the bulk arrows) for third pressurization cycles at $295 \mathrm{~K}$. (e) and (f) Pressure dependencies of the electrical resistance for two $\mathrm{HgTe}_{1-x} \mathrm{~S}_{x}$ samples with (e) $x=0.05$ and $(\mathrm{f}$ ) $x=0.1$ for two pressurization and decompression cycles. The thin arrows near the curves point out the directions of pressure variation. One can see the excellent data reproducibility for the sample with (e) $x=0.05$, whereas the sample with (f) $x=0.1$ showed a notable difference in the curves between the third and six cycles.

topological insulator under biaxial/uniaxial compressive deformation; ${ }^{72,110-112}$ interesting topological features were proposed for $\mathrm{HgTe}_{1-x} \mathrm{~S}_{x}$ too. ${ }^{109}$

We investigated variations in the thermopower and the electrical resistance of the solid solutions of $\operatorname{HgTe}_{1-x} \mathrm{~S}_{x}(x=0,0.05,0.10$, and 0.15 ) under applied high pressure across the electronic and structural phase transitions for multiple pressurizationdecompression cycles. An abrupt rising in both the electrical resistance and the thermopower above 1-3 GPa [Figs. 1(d)-1(f), 2, and $3(\mathrm{a})$ ] corresponded to the transition to the semiconductor cinnabar phase. ${ }^{98-100}$ The pressure dependencies of the thermopower for binary $\mathrm{HgTe}$ exhibited a pronounced and reproducible bend at about 0.6-0.9 GPa [Fig. 3(b)], i.e., in the sphalerite-type phase. As seen from [Figs. 2(b)-2(d) and 3(c)], the sulfur substitution shifted this feature from the $p$ - to $n$-type region, thereby demonstrating a relative enhancement of the electron partial conductivity.

Generally, the Seebeck coefficient of all $\mathrm{HgTe}_{1-x} \mathrm{~S}_{x}$ samples exhibited the colossal variations under applied pressure, which included a reversible $n-p-n$ double sign inversion of the electrical conductivity (Figs. 2 and 3). With the increase in both the sulfur content and the pressure cycle number, the $n-p$ conductivity inversion point shifted to the higher pressures (Figs. 2 and 3). The first $n-p$ sign inversion took place in $\mathrm{HgTe}$ at about $0.5-0.8 \mathrm{GPa}$, i.e., before the bend in the curves [Figs. 1(d)-1(f), 2, and 3(a)]; in $\mathrm{HgTe}_{0.95} \mathrm{~S}_{0.05}$ for the first pressure cycle, it slightly shifted to $0.9 \mathrm{GPa}$. The $n-p$ inversion in $\mathrm{HgTe}$ was reversible and reproducible for the subsequent pressurization cycles [Fig. 3(b)], whereas for the second and subsequent pressure cycles in $\mathrm{HgTe}_{0.95} \mathrm{~S}_{0.05}$ and for all cycles in $\mathrm{HgTe}_{0.90} \mathrm{~S}_{0.10}$ and $\mathrm{HgTe}_{0.85} \mathrm{~S}_{0.15}$ samples, no $n-p$ inversion in the sphalerite-type phase was observed; the electrical conductivity turned to the $p$-type only after the structural phase transition to the cinnabar phase [Fig. 1(b)]. The maxima of the pressure dependencies of the thermopower and the resistance at about $\sim 2-5 \mathrm{GPa}$ corresponded to the pure cinnabar phase, whose bandgap for binary $\mathrm{HgTe}$ was found to be as $E_{g} \sim 0.6-$ $0.7 \mathrm{eV} .^{82,88,99,100}$ The following progressive decreasing in both the Seebeck coefficient and the resistance in the cinnabar phase with pressure reflected the bandgap narrowing. ${ }^{82,99,100}$ Remarkably, the 

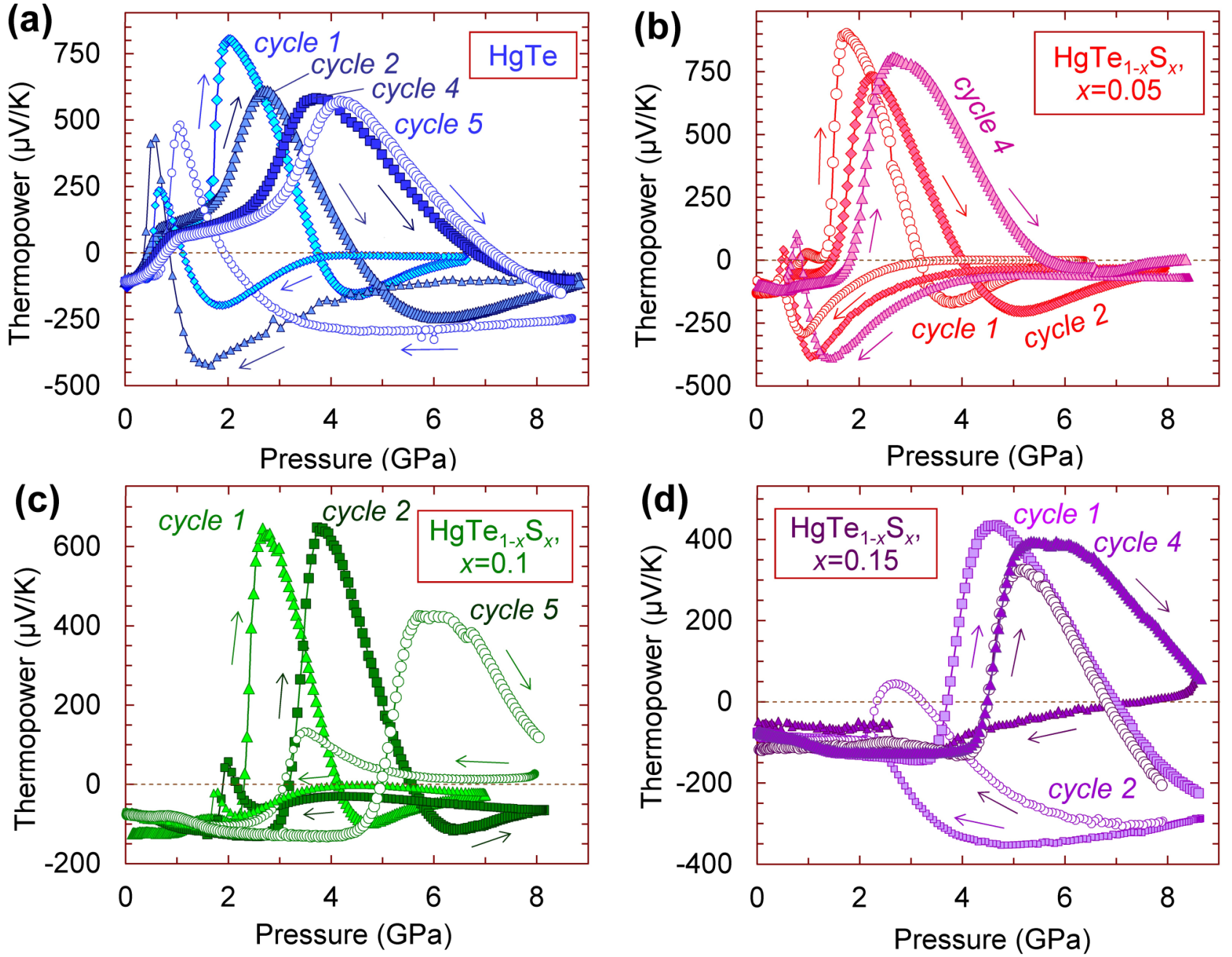

FIG. 2. Pressure dependencies of the thermoelectric power (the Seebeck coefficient) of $\mathrm{HgTe}_{1-x} \mathrm{~S}_{x}$ single crystals with (a) $x=0$, (b) $x=0.05$, (c) $x=0.1$, and (d) $x=0.15$ for selected pressurization and decompression cycles at $295 \mathrm{~K}$. The thin arrows near the curves point out the directions of pressure variation. These plots show a tendency to the reproducibility of the dependencies for multiple pressure cycling.

thermopower in the cinnabar phase of all $\mathrm{HgTe}_{1-x} \mathrm{~S}_{x}$ samples first inverted its sign from the $p$ - to $n$-type above $3-7 \mathrm{GPa}$ and then, inverted its pressure derivative [Figs. 2 and $3(\mathrm{a})$ ].

The pressure dependences of the thermopower for $\mathrm{HgTe}_{1-x} \mathrm{~S}_{x}$ samples, for the exception of the one with $x=0.15$, exhibited the smooth inflection points in the negative region at about $4-6 \mathrm{GPa}$ (Fig. 2). These features may be addressed to the onset of the phase transition to the NaCl-type phase [Fig. 1(c)]. However, the electrical resistance curves of these samples did not pronounce changes in these pressures [Fig. 1(d)]. Only for one of them, namely, with $x=0.05$, the resistance value notably dipped near above $5 \mathrm{GPa}$ that could be linked to this transition. Earlier studies found that the $\mathrm{NaCl}$ phase in $\mathrm{HgTe}$ is metallic and has a $p$-type conductivity. ${ }^{106}$ Above 4-6 GPa, after the smooth negative extremum, the Seebeck coefficients of these three $\mathrm{HgTe}_{1-x} \mathrm{~S}_{x}$ samples showed an apparent tendency to decreasing of their absolute values, followed by an $n-p$ sign inversion (Fig. 2). Therefore, we can infer that in the pressure range investigated these three samples probably only partly transformed to the $\mathrm{NaCl}$-type structure, and hence, the reproducible double $n-p-n$ conductivity inversions observed in all $\mathrm{HgTe}_{1-x} \mathrm{~S}_{x}$ samples [Fig. 3(a)] corresponded to the sphalerite and cinnabar phases.

Although the pressure-driven variations in the thermoelectric power and electrical resistance of $\mathrm{HgTe}$ and $\mathrm{HgTe}_{1-\mathrm{x}} \mathrm{S}_{\mathrm{x}}$ samples were well reproduced for successive pressure cycles, they showed a significant shift of the features to the higher pressures with the cycle number. We can address this effect to residual strains and plastic deformation of the original single-crystalline crystals. HgTe 

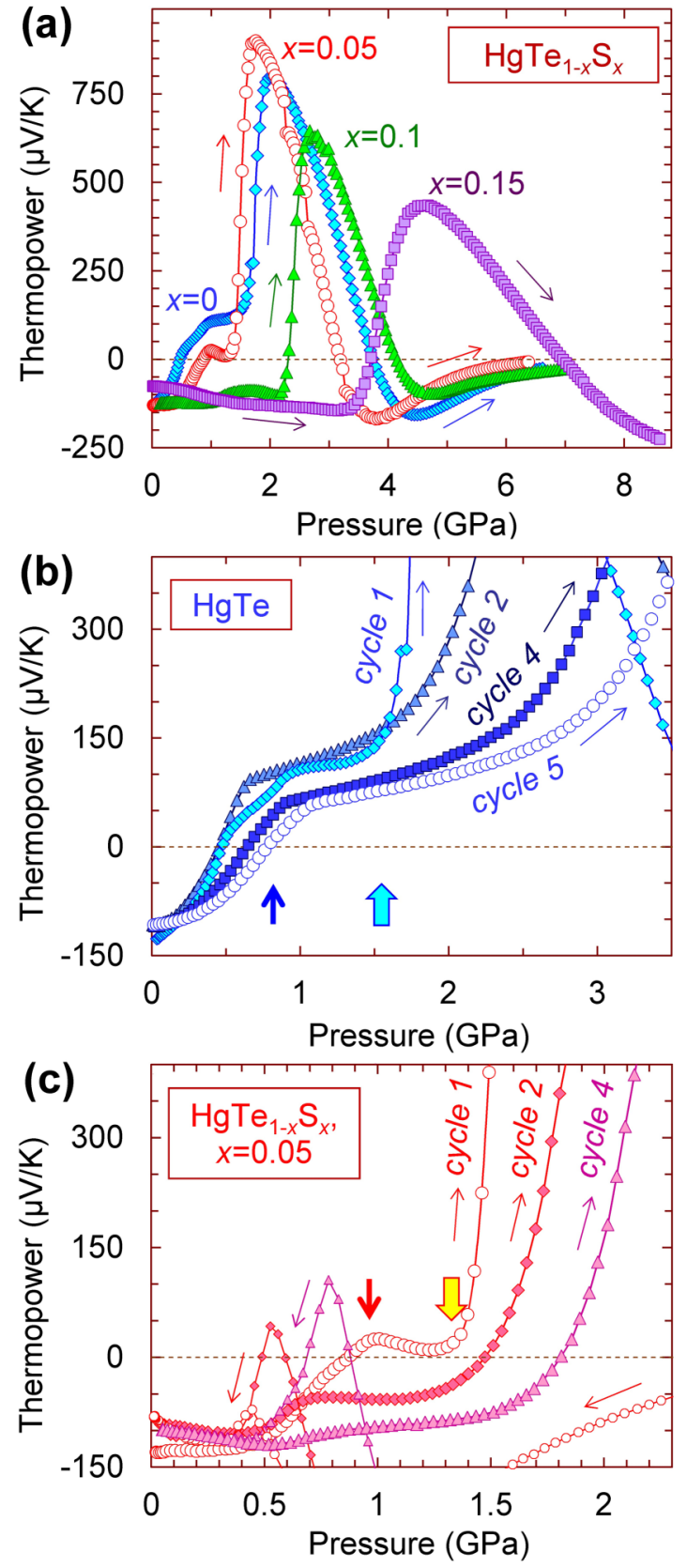

FIG. 3. Selected pressure dependencies of the thermoelectric power (the Seebeck coefficient) for $\mathrm{HgTe}_{1-x} \mathrm{~S}_{x}$ single crystals replotted from Fig. 2. The thin arrows near the curves point out the directions of pressure variation. (a) For the first pressurization cycle for all $\mathrm{HgTe}_{1-x} \mathrm{~S}_{x}(x=0,0.05,0.1$, and 0.15$)$ samples. (b) For the HgTe sample in the pressure range up to $3.5 \mathrm{GPa}$. The thin vertical arrow points out the bend for the first cycle, which could be related to the electronic transition in the sphalerite phase. The bulk arrow points out the structural phase transition from the sphalerite to the cinnabar phase for the first cycle. (c) For the sample with $x=0.05$ in the pressure range up to $2.3 \mathrm{GPa}$. The notations are the same as in (b). As seen the feature at about $1 \mathrm{GPa}$ is suppressed with pressure cycling. and $\mathrm{HgTe}_{1-\mathrm{x}} \mathrm{S}_{\mathrm{x}}$ are rather soft materials, and under an applied high pressure, they can be not only elastically compressed but plastically deformed too. In addition, an earlier study of similar HgSe compounds discovered that in a vicinity of a zinc-blende $\rightarrow$ cinnabar phase transition, these fragile materials become highly plastic and can be significantly deformed. ${ }^{113}$ Furthermore, this earlier study also found that these plastically deformed samples recovered after a high hydrostatic pressure of $2 \mathrm{GPa}$ are characterized by enhanced microhardness values. ${ }^{113}$ Thus, the shifts of the transition point to the higher pressures with the cycle number we observed in all $\mathrm{HgTe}_{1-x} \mathrm{~S}_{x}$ samples can be explained by the above hardening of the samples after their successive plastic deformation.

\section{B. $\mathrm{PbTe}$}

At ambient conditions, lead telluride, $\mathrm{PbTe}$, crystallizes in the cubic rock salt-type $(\mathrm{NaCl})$ structure [Fig. 1(c)]. ${ }^{114}$ Under applied high pressure above 5.5-7 GPa, this phase undergoes a phase transition to an orthorhombically distorted structure of Pnma symmetry. ${ }^{56-59,115,116}$ Although this transition has been discovered a long time ago, ${ }^{76,117-122}$ and high-pressure effects on the electronic band structure of $\mathrm{PbTe}$ and its parameters were investigated in a plenty of studies, ${ }^{48,49,51,53,54,63,64,123-126}$ the pressure-driven reconstructions of the band structure of $\mathrm{PbTe}$ in both the $\mathrm{NaCl}$ and Pnma phases remain obscure to the date. As it was briefly mentioned in Sec. I paragraph, the direct bandgap of $\mathrm{PbTe}$ in the $\mathrm{NaCl}$ phase, $E_{g}=0.3 \mathrm{eV}$, was established to decrease linearly with the applied pressure with a coefficient of about $-(70 \div 90) \mathrm{meV} /$ GPa. ${ }^{47-54}$ This fact suggests that the bandgap should be eventually closed at about 3-4 GPa, ${ }^{49,63,64}$ i.e., well before the phase transition point of $5.5-7 \mathrm{GPa},{ }^{56-59}$ at which, a small bandgap is re-opened [Fig. 4(a)]. ${ }^{60,61,127}$ Experimentally, an electronic state that corresponds to the minimal or zero value of the direct bandgap was indeed observed at about $3 \mathrm{GPa}^{55}$ The above-discussed alterations in the band structure should result in significant modifications in the electronic topology. Recent band structure calculations proposed for $\mathrm{PbTe}$ a transition from a trivial insulator to a topological one at $\sim 3.4-4.8 \mathrm{GPa}^{46,49,63,64,128,129}$ The electrical conductivity $(\sigma)$ of $\mathrm{PbTe}$ was found to enhance greatly in this "gapless" state under conservation of significant magnitudes of its Seebeck coefficient $(S) ;{ }^{62}$ this resulted in a colossal improvement of its power factor $\left(S^{2} \sigma\right) .{ }^{62}$ Notably that PbSe, which adopts the same NaCl-type structure and has the same direct bandgap, also showed an enhancement of its thermoelectric properties at $2-3 \mathrm{GPa} .{ }^{30}$

We investigated the electronic transport properties of several $\mathrm{PbTe}$ single crystals under pressure cycling up to $9 \mathrm{GPa}$ across the electronic and structural phase transitions (Fig. 4). The electrical resistance of $\mathrm{PbTe}$ progressively diminished with pressure and showed smooth features at about 3 and 7 GPa [Fig. 4(a)]. In one previous work that reported a pressure dependence of electrical resistivity for heavily doped $\mathrm{PbTe}$ crystals, a pronounced jump in the resistivity value near about $2 \mathrm{GPa}$ was observed. ${ }^{130}$ This feature could be linked to the above-discussed electronic transition. Probably, in this case, the doping enhanced the signatures of this transition. However, other studies for undoped PbTe crystals did not detect any remarkable changes across this transition. ${ }^{131}$ All the investigated crystals of $p$-type $\mathrm{PbTe}$ inverted their original 

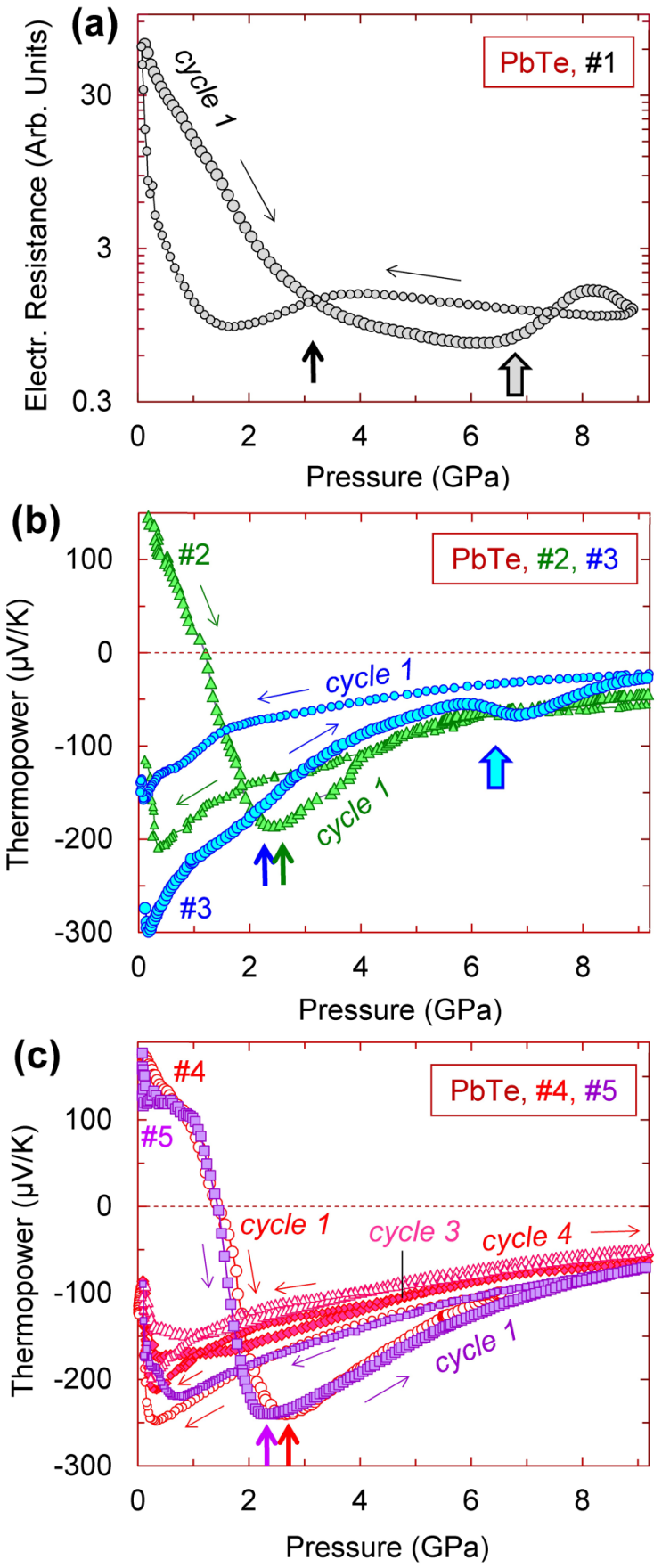

FIG. 4. Pressure dependencies of (a) the electrical resistance and (b) and (c) the thermoelectric power (the Seebeck coefficient) of five crystals of PbTe (labeled as 1-5) for several pressurization and decompression cycles at $295 \mathrm{~K}$. The thin arrows near the curves point out the directions of pressure variation. The thin vertical arrows point out the electronic transition in the rock salt phase. The bulk arrows point out the structural $\mathrm{NaCl} \rightarrow P n m a$ phase transition at $\sim 6$ $7 \mathrm{GPa}$. (c) Samples 4 and 5 were cut from the same bulk crystal and their dependencies are nearly identical. dominant electrical conductivity to $n$-type and reached the maximal negative Seebeck coefficients at $\sim 2.5 \mathrm{GPa}$ [Figs. 4 (b) and 4 (c)]. The structural $\mathrm{NaCl} \rightarrow$ Pnma phase transition was noticeable on some thermopower curves as a minor dip at about 6-7 GPa [Fig. 4(b)]. Remarkably that the electronic transition resulted in much more significant variations in the thermoelectric power, compared to those linked to the $\mathrm{NaCl} \rightarrow$ Pnma structural phase transition. ${ }^{56-59}$

We verified for several $p$-PbTe samples that the pressuredriven $p-n$ inversion is irreversible, and the successive pressure cycles correspond already to the $n$-type conductivity [Fig. 4(c)]. For comparison, we measured the thermopower of as-grown $n$-type single crystal of $\mathrm{PbTe}$ (sample 3) and observed a similar pressure behavior but with a more distinct dip in a vicinity of the structural transition [Fig. 4(b)]. Our findings suggest that applied pressure or stress of several GPa can dramatically alter the electronic properties of $p$-type PbTe crystals and even turn them into $n$-type conductors.

\section{C. $\mathrm{Bi}_{2} \mathrm{Te}_{3}$}

At normal conditions, $\mathrm{Bi}_{2} \mathrm{Te}_{3}$ crystallizes in a tetradymite layered structure (rhombohedral space group $R \overline{3} m$ No. 166) [Figs 5(a) and 5(b)] that is characterized by a low thermal conductivity. ${ }^{132}$ Note here that high-pressure high-temperature synthesis can stabilize other metastable polymorphs at ambient conditions. ${ }^{133-135}$ An unusual band structure topology of $\mathrm{Bi}_{2} \mathrm{Te}_{3}$ allows to implement a topological insulator state with a small bulk bandgap of about $E_{g} \sim 0.15 \mathrm{eV} .^{33,136,137}$ A combination of the low thermal conductivity with the topological insulator state (which provides a high power factor, $S^{2} \sigma$ ) can explain the high room-temperature thermoelectric performance parameters of $\mathrm{Bi}_{2} \mathrm{Te}_{3}{ }^{138-145}$ It was found that the applied pressure of about 2-4 GPa induces the number of remarkable phenomena. They included, for example, 2D electronic topological Lifshitz transition, ${ }^{45}$ the emergence of superconductivity, ${ }^{146-148}$ giant enhancement of thermoelectric power factor, ${ }^{149,150}$ and also spectacular changes in the bulk mechanical, ${ }^{45,151,152}$ electronic, ${ }^{153-155}$ vibrational, and other properties. ${ }^{156-159}$ Above 6-8 GPa, the original rhombohedral phase transforms to a monoclinic $\mathrm{C} 2 / \mathrm{m}$ structure. $^{22,146,148,151,157,158,160-165}$

Here, we investigated a possibility of pressure-driven $p-n$ conductivity switching in $\mathrm{Bi}_{2} \mathrm{Te}_{3}$. We measured the Seebeck coefficient and the electrical resistance of an undoped $p$-type crystal with a low carrier concentration as $p \sim 5 \times 10^{17} \mathrm{~cm}^{-3}$ across the electronic and structural phase transitions [Fig. 5(c) and 5(d)]. One can see that at a pressure of about $3 \mathrm{GPa}$, which corresponded to the above 2D electronic topological transition, ${ }^{45}$ the dominant electrical conductivity of $\mathrm{Bi}_{2} \mathrm{Te}_{3}$ can be reversibly inverted to the $n$-type [Fig. 5(d)]. The electrical resistance curve exhibited a bend near this pressure [Fig. 5(c)]. In previous works, a dip on pressure dependencies of the thermopower without $p-n$ inversion was also observed at similar pressures for $p$-type $\mathrm{Bi}_{2} \mathrm{Te}_{3}$ crystals with the higher carrier concentrations above $\sim 10^{18} \mathrm{~cm}^{-3}$. 149,150 These findings suggest that this electronic transition results in a rather limited enhancement of the $n$-type partial conductivity, and hence, such a double $p-n-p$ inversion in the vicinity of $3 \mathrm{GPa}$ can be observable only in high-quality undoped $p$-type crystals. This result is in 

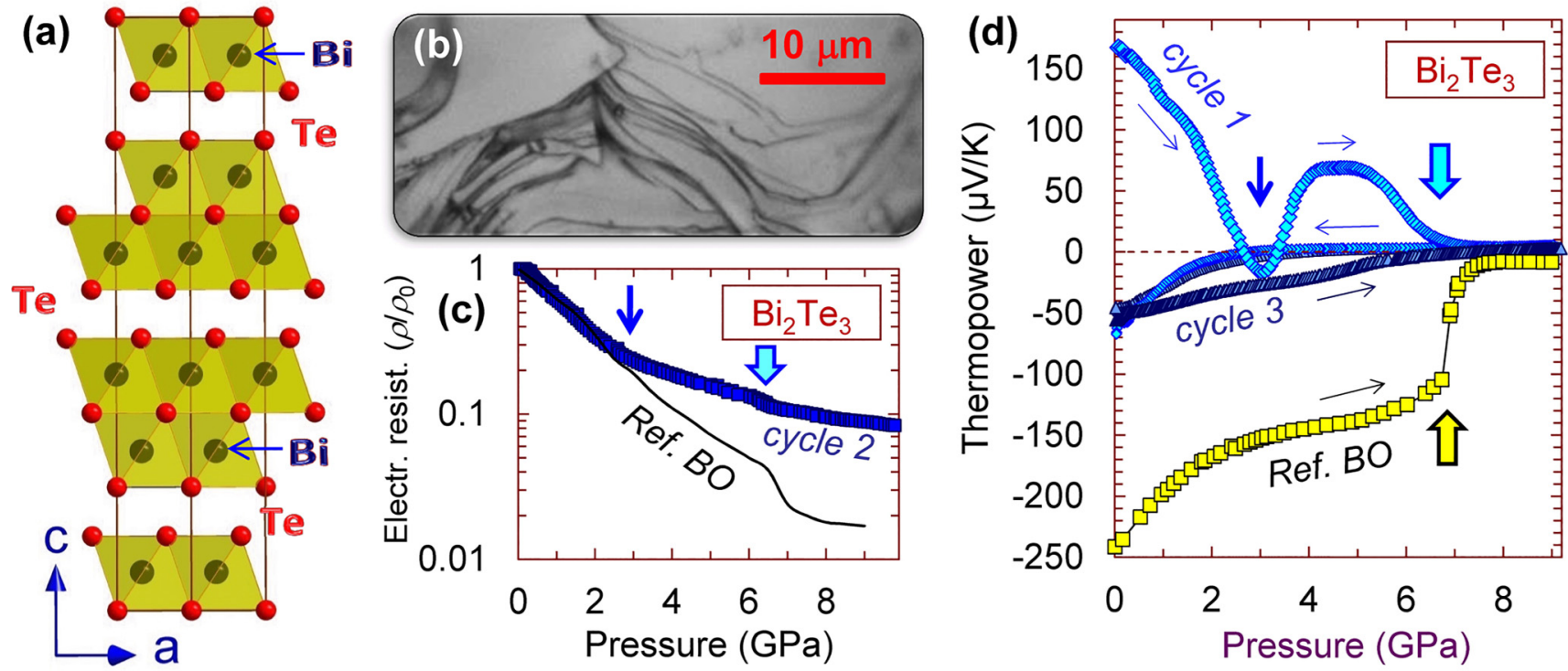

FIG. 5. (a) and (b) Room-temperature crystal structure and a photograph clearly showing the layered structure of $\mathrm{Bi}_{2} \mathrm{Te}_{3}$ crystals. (c) and (d) Pressure dependencies of the electrical resistance and the thermoelectric power (the Seebeck coefficient) of $\mathrm{Bi}_{2} \mathrm{Te}_{3}$ crystals at $295 \mathrm{~K}$. The thin arrows near the curves point out the directions of pressure variation. The thin vertical arrows point out the electronic transition in the rhombohedral phase, investigated, for example, in Ref. 45. The bulk arrows point out the structural phase transition from the original rhombohedral phase to the monoclinic $\mathrm{C} 2 / \mathrm{m}$ structure at about $7 \mathrm{GPa}$. For comparison, we plotted in (c) and (d), the pressure dependencies of the electrical resistance and the thermopower for $n$-type $\mathrm{Bi}_{2} \mathrm{Te}_{3}$ single crystals from Ref. 167 (labeled as Ref. $B O$ near the curves).

contrast to the above cases of $\mathrm{HgTe}$ and $\mathrm{PbTe}$, in which the electronic transitions led to the more dramatic changes in the conductivity [Figs. 3(b), 4(b), and 4(c)]. On the phase transition to the C2/ $m$ phase at $\sim 6-8 \mathrm{GPa},{ }^{22,146,148,151,157-163,166}$ the residual minor bandgap of $\mathrm{Bi}_{2} \mathrm{Te}_{3}$ is abruptly closed, and its electrical resistance drops [Fig. 5(c)]. We found that the conductivity in the $\mathrm{C} 2 / \mathrm{m}$ phase can be both of $p$ - and $n$-types [Fig. 5(d)], thereby indicating that this phase is rather a semimetal than a metal. We also established that the samples recovered from the structural phase transition demonstrate a strong preference to the $n$-type conductivity. Thus, alike $p$-type $\mathrm{PbTe}$, an applied moderate pressure can irreversibly turn $\mathrm{Bi}_{2} \mathrm{Te}_{3}$ to an $n$-type conductor.

\section{DISCUSSION}

\section{A. The high-pressure effects on the thermopower of the tellurides}

The Seebeck coefficients of the topological tellurides investigated demonstrated the colossal variations under applied moderate high pressure, including the controlled inversions of the conductivity type (Figs. 2-5). The most spectacular changes in the electronic properties were observed in the vicinities of the electronic topological transitions in the conventional normal-pressure crystalline phases. Since these features were not linked to any crystal-structure reconstructions, they had to come out from the modifications of their band structures.

At normal conditions, the valence and conduction bands in the conventional zinc-blende phase of $\mathrm{HgTe}$ are a bit overlapped at the $\Gamma$ point of the Brillouin zone, suggesting a gapless semiconductor state. $^{70,73,168,169}$ In particular, $s$-like minimum of the conduction band with $\Gamma_{6}$ symmetry lies below the $p$-like maximum of the valence band with $\Gamma_{8}$ symmetry. ${ }^{73,168,169}$ A band with $\Gamma_{7}$ symmetry lies below the band with $\Gamma_{6}$ symmetry because of a large spin-orbit splitting. ${ }^{71,73,168-170}$ Under applied pressure, the $\Gamma_{6}$ band (which corresponds to a "light" hole band) was found to move upward in the energy, and, at a certain pressure, it should cross the $\Gamma_{8}$ band (which corresponds to a "heavy" hole band), thereby inverting the band structure. $^{79,80}$ Then, at a higher pressure, a semiconductor gap between the $s$ - and $p$-bands is expected to open. The above upshift of the "light" band of mobile holes should stimulate a transfer of the holes carriers to this band, and hence, a $p$-type partial conductivity should be notably enhanced under pressure. This can explain the abrupt $n-p$ inversion observed in binary HgTe [Fig. 3(b)]. Previous works reported that sulfur substitution in HgTe increases the concentration of electrons and stabilizes its $n$-type conductivity. ${ }^{105}$ As we observed for the compound with $x=0.05$, the $n-p$ inversion is still observable about $1 \mathrm{GPa}$ but it is not robust and is suppressed with the subsequent pressure cycles [Fig. 3(c)]. This is likely due to the formation of donor-type defects in the samples (e.g., Te vacancies) that stabilized the $n$-type conductivity. In these samples with the higher sulfur content, this $n-p$ inversion degenerated into a minor anomaly on the curves (Fig. 2).

Recent Hall-coefficient studies of the zinc-blende phase of $\mathrm{HgTe}$ reported a pressure-driven decrease in an effective carrier concentration and an increase in their mobility. ${ }^{106}$ These findings confirmed a pressure-driven reduction of the overlap of the valence and conduction bands, thereby suggesting that at a certain pressure they should be completely separated. ${ }^{79,80,106}$ Note here that the 
band structure of HgTe is very sensitive to many factors, like small strains or defects, and hence, the fine details of the band structure can vary a bit from sample to sample. ${ }^{168}$ The rising in the electrical resistance with the pressure we observed in the zinc-blende phase of HgTe [Fig. 1(d)] could be explained by the gradual reduction of the bands overlap. However, instead of a bandgap opening in the zinc-blende phase, HgTe undergoes the structural transformation to the hexagonal cinnabar-type structure with the inherent large bandgap [Figs. 1(b)-1(d)]. ${ }^{7}$

The pressure dependencies of the electrical resistance and the thermopower of $\mathrm{HgTe}_{1-x} \mathrm{~S}_{x}$ indicated that above the phase transition point of $1-4 \mathrm{GPa}$, the cinnabar phase of all the samples is a $p$-type semiconductor, in agreement with previous works. ${ }^{80,101,103,106}$ The thermopower in the cinnabar phase of $\mathrm{HgTe}_{1-x} \mathrm{~S}_{x}$ can be well described by a formula for an intrinsic semiconductor with a two-band electrical conductivity as follows: ${ }^{171}$

$$
\begin{array}{r}
S=-\frac{k}{|e|}\left[\frac{\sigma_{n}-\sigma_{p}}{\sigma_{n}+\sigma_{p}} \frac{E_{g}}{2 k T}+\left(r_{n}+\frac{5}{2}\right) \frac{\sigma_{n}}{\sigma_{n}+\sigma_{p}}\right. \\
\left.-\left(r_{p}+\frac{5}{2}\right) \frac{\sigma_{p}}{\sigma_{n}+\sigma_{p}}-\frac{3}{4} \ln \frac{m_{p}^{*}}{m_{n}^{*}}\right],
\end{array}
$$

where $k$ is Boltzmann's constant, $e$ is the electron charge, $T$ is the temperature, $\sigma_{n}\left(\sigma_{p}\right)$ is the partial conductivity of electrons (holes), and $r_{n}\left(r_{p}\right)$ and $m_{n}^{*}\left(m_{p}^{*}\right)$ are the scattering parameter and the effective mass of the density of states of electrons (holes), respectively.

Thus, the thermopower variation in the cinnabar phase of $\mathrm{HgTe}_{1-x} \mathrm{~S}_{x}$ samples is mainly determined by changes in the bandgap and in the partial conductivities [Eq. (1)]. The bandgap of the cinnabar phase of HgTe just after the completion of the transition was reported to be about $0.6-0.7 \mathrm{eV} ;{ }^{82,88,99,100}$ its pressure coefficient was established as $\mathrm{d} E_{g} / \mathrm{d} P \approx-(120 \div 153) \mathrm{meV} /$ $\mathrm{GPa}^{82,99,100}$ In a cinnabar phase of $\alpha$-HgS, a bandgap at ambient conditions is much larger as $E_{g}=2.2 \mathrm{eV}$, and hence, the sulfur substitution should notably increase the above bandgap in $\operatorname{HgTe}_{1-x} \mathrm{~S}_{x}$ samples. Qualitatively, it was seen from the rising in the maximum resistance values at about $2-4 \mathrm{GPa}$ in the cinnabar phases of $\operatorname{HgTe}_{1-x} \mathrm{~S}_{x}$ with the $x$ content [Fig. 1(d)]. At the same time, the maxima of the Seebeck coefficients in the cinnabar phases at about 2-5 GPa, on the contrary, diminished with the $x$ content [Fig. 3(a)]. Therefore, in accordance with Eq. (1), these findings directly pointed out the enhancement of the $n$-type conductivity in the sulfur-substituted samples with the $x$ content. Interestingly to note that above 3-7 GPa, the conductivity in the cinnabar phases inverted from $p$ - to $n$-type [Fig. 3(a)]. This effect might be linked, for instance, to shifting down the "light" hole band, followed by a charge transfer to the "heavy" hole band, i.e., be opposite to that discussed above for the zinc-blende phase.

$\mathrm{PbTe}$ is a direct-gap semiconductor whose gap of about $0.3 \mathrm{eV}$ lies at the $L$ point of the Brillouin zone. ${ }^{52,172,173}$ The top of its valence band presents a band of "light" and high-mobile holes, ${ }^{52,172,173}$ whereas a band of "heavy" and less-mobile holes lies at the $\Sigma$ point of the Brillouin zone, well below the above "light" band, and hence, it does not usually contribute to its electrical conductivity. ${ }^{127}$ The pronounced $p-n$ inversion observed in PbTe at $\sim 1-2 \mathrm{GPa}$ [Figs. 4(b) and 4(c)] cannot come out from the pressure-driven bandgap narrowing in the NaCl-type phase. ${ }^{47-54}$ Hence, one can propose that the applied pressure shifted up the level of the "heavy" hole band, relative to the "light" band, and stimulated a redistribution of the holes between these two bands. It should lead to a strong suppression in the partial hole conductivity in $\mathrm{PbTe}$ since the "heavy" holes are much less mobile, than the "light" ones. It could explain the $p-n$ sign inversion at $\sim 1-2 \mathrm{GPa}$ [Figs. 4(b) and 4(c)]. Earlier investigations of p-type PbTe showed that the carrier mobility $\mu$ first increases with pressure up to $\sim 2-$ $3 \mathrm{GPa}$ because of the narrowing of its direct bandgap $\left(\mu \sim 1 / E_{g}\right)$ but then decreases with further pressurization. ${ }^{55}$ Theoretical calculations found that the bandgap closure in PbTe at $\sim 3 \mathrm{GPa}$ leads to the band inversion, ${ }^{64,128,129}$ which is driven by an enhanced $s p$-hybridization, ${ }^{129}$ followed by increasing the band overlapping (negative gap) under further compression. ${ }^{64}$ These effects turn $\mathrm{PbTe}$ into a topological insulator. ${ }^{46,49,64,128,129}$ The negative extremum on the thermopower curves likely corresponded to this transition [Figs. 4(b) and 4(c)]. The above-discussed band structure reconstructions seem to be reversible. Hence, we can address the irreversibility of the $p-n$ inversion in PbTe to either (i) the conservation of the residual strains in the samples, which could keep the position of the "heavy" hole band on the top of the valence band, or (ii) the formation of the large number of donor-type structural defects in the compressed samples, which could shift the charge balance to the $n$-type.

In $\mathrm{Bi}_{2} \mathrm{Te}_{3}$, we found that the above-discussed $2 \mathrm{D}$ electronic topological Lifshitz transition at about $3 \mathrm{GPa}^{45}$ enhanced the $n$-type partial conductivity only in the limited pressure region [Fig. 5(d)] and seemed to be reversible. As known from the literature, ${ }^{174-179}$ the top of the valence band of bulk $\mathrm{Bi}_{2} \mathrm{Te}_{3}$ crystals consists of a six-valley band of "light" holes and a lying below the band of "heavy" holes, whose effective masses and mobility values essentially differ. One can propose for $\mathrm{Bi}_{2} \mathrm{Te}_{3}$ the similar band structure reconstructions as in the above-discussed case of $\mathrm{PbTe}$. Thus, the abrupt collapse of the $p$-type conductivity in $\mathrm{Bi}_{2} \mathrm{Te}_{3}$ at $\sim 3 \mathrm{GPa}$ [Fig. 5(d)] could be likely caused by a redistribution of the charge carriers between the groups of the "light" and "heavy" hole bands. It is worthy to mention that a recent hydrostatic-pressure study of the Seebeck coefficient of $n$-type $\mathrm{Bi}_{2} \mathrm{Te}_{3}$ single crystals disclosed a spectacular $n-p-n$ double sign inversion just prior to the structural phase transition to the $C 2 / m$ phase. ${ }^{163}$ This finding hints at the existence of one more electronic transition in $\mathrm{Bi}_{2} \mathrm{Te}_{3}$ that enhances the $p$-type partial conductivity and which can be potentially observable in high-quality $n$-type crystals. Recall here that in this work, the thermopower curves for the $n$-type samples did not show this feature [Fig. 5(d)]. The irreversible $p-n$ conductivity inversion observed in the sample recovered from the structural $R \overline{3} m \rightarrow C 2 / m$ phase transition [Fig. 5(d)] is likely due to the appearance of the sizable number of peculiar structural defects of the $n$-type that change the charge balance in the sample.

\section{B. Potential innovative applications of the tellurides}

The spectacular inversions of the electrical conductivity type observed here, which are reversible in HgTe (Figs. 2 and 3) and 

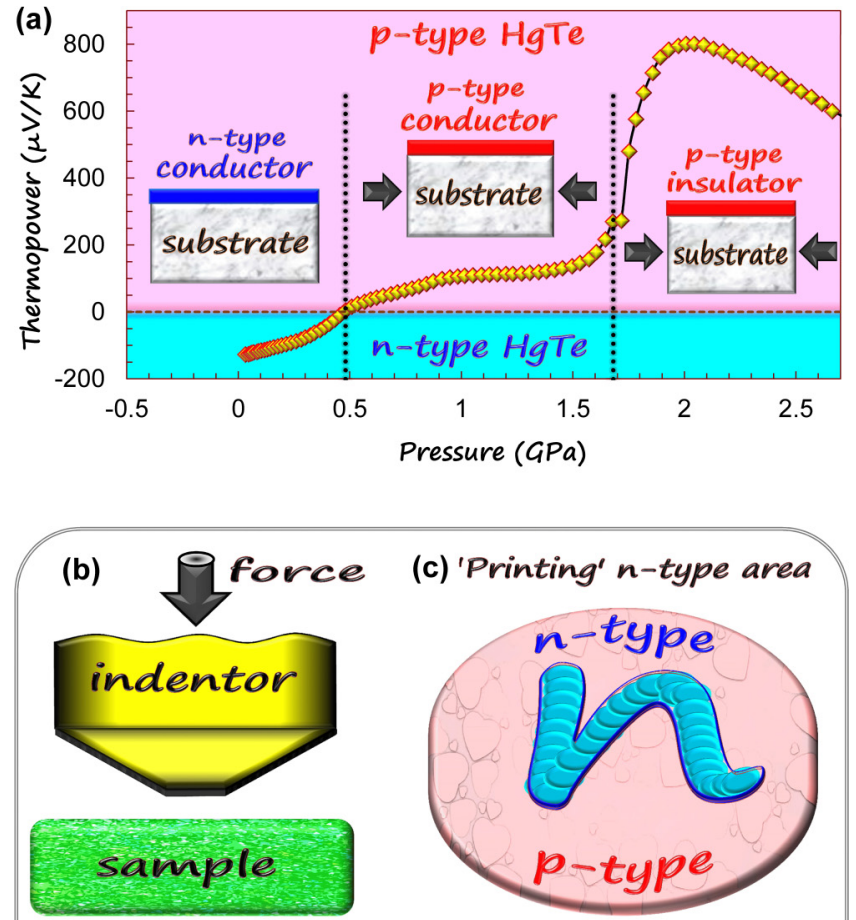

(c) 'Printing' n-type area

(d) above $4 \mathrm{GPa}$

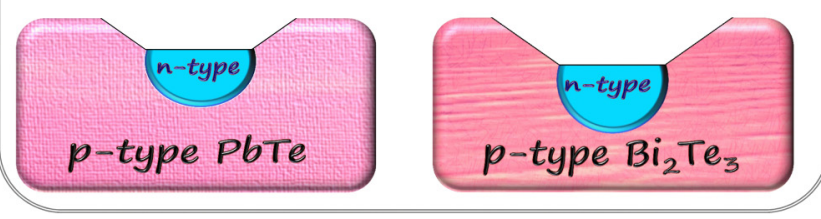

FIG. 6. Examples of potential industrial applications of the pressure-driven effects in $\mathrm{HgTe}, \mathrm{PbTe}$, and $\mathrm{Bi}_{2} \mathrm{Te}_{3}$. Plot (a) depicts the pressure dependence of the thermopower for $\mathrm{HgTe}$ from Fig. 2(a) and illustrates that by applying small controlled stresses (e.g., generated in a thin film of HgTe deposited on the substrate), one can reversibly switch its electronic state. Possible electronic states of HgTe include: (i) $n$-type conductor and (ii) $p$-type conductor in the normalpressure sphalerite phase, and (iii) $p$-type insulator in the high-pressure cinnabar phase [Figs. 1-3]. Plots (b)-(e) illustrate that by applying a hard indenter tool (that can generate high stresses) to sample surface (b), one can "print out" areas with desirable conductivity type. Plots (d) and (e) depict anticipated depth profiles of $n$-type conductivity area, which can be "printed out" on the surface of $p$-type $\mathrm{PbTe}$ and $p$-type $\mathrm{Bi}_{2} \mathrm{Te}_{3}$ by applying stresses above 4 and $9 \mathrm{GPa}$, respectively.

irreversible in PbTe [Figs. 4(a) and 4(b)] and $\mathrm{Bi}_{2} \mathrm{Te}_{3}$ [Fig. 5(d)], look highly promising for potential applications in the industry. For instance, they could be implemented in next-generation nanoelectronic devices, operating with applying tunable stresses. Here, we can propose a pair of the simplest applications of these effects. It is known that high strains (which simulate applied stresses) can be generated in thin films deposited on the substrate, for instance, because of "misfit" lattice parameters in selected pair of the film and the substrate. ${ }^{12,13,180-182}$ These strains allow us to stabilize high-pressure phases at normal conditions, as it was implemented, e.g., in $\mathrm{PbTe} .^{12,13}$ Hence, one can propose that the high-pressure effects on the electronic properties of HgTe [Figs. 2(a) and 3(a)] could be potentially implemented in a thin film deposited on the substrate, in which, generation of small tunable or constant strains (simulating stresses of up to $3 \mathrm{GPa}$ and higher) would be somehow possible. As illustrated in Fig. 6(a), these strains or applying stresses can reversibly switch between three different electronic states, namely, the $n$-type and $p$-type conductors in the conventional sphalerite phase up to $1.7 \mathrm{GPa}$ that is characterized by the high electrical conductivity [Fig. 1(d)] and the p-type insulator, which is linked to the cinnabar-type structure above 1.7 GPa (Fig. 1).

The same approach as described above can be applied to $p$-type $\mathrm{PbTe}$ films too, in which, an applied stress of about $2 \mathrm{GPa}$ can induce the $p-n$ conductivity inversion [Figs. 4(b) and 4(c)]. As found in recent high-pressure studies of Ge-doped silicon, such $p-n$ sign inversions, which are not accompanied by crystal-structure changes, can be reversible if the applied pressure does not go beyond the negative extremum point, ${ }^{7}$ i.e., $2 \mathrm{GPa}$ for $\mathrm{PbTe}$; whereas the higher stresses led to notable irreversible changes in a defect structure of these Si:Ge samples. ${ }^{7}$ In this study, we applied to the PbTe crystals the high pressures well above $2 \mathrm{GPa}$ and found that the $p-n$ switching is irreversible. Although on the decompression cycles below $\sim 0.5 \mathrm{GPa}$, a tendency to a partial recovery of the original characteristics of the $\mathrm{PbTe}$ samples was still noticeable [Figs. 4(b) and 4(c)].

The irreversible $p-n$ inversions observed in PbTe [Figs. 4(b) and $4(\mathrm{c})$ ] and $\mathrm{Bi}_{2} \mathrm{Te}_{3}$ [Fig. 5(d)] suggest that these semiconductors can be potentially utilized in technologies that operate applying stresses to "print out" areas with a certain conductivity type [Fig. 6(c)]. For instance, it could be accomplished with the assistance of electronically controlled hard tips of designed shapes [Fig. 6(b)] that can produce high stresses on the sample surface under applied loads of less than a few grams. ${ }^{181}$ Using this method, one can "print out" $n$-type semiconductor areas on surfaces of p-type bulk samples of $\mathrm{PbTe}$ and $\mathrm{Bi}_{2} \mathrm{Te}_{3}$ [Figs. 6(c)-6(e)].

\section{CONCLUSIONS}

In this work, we investigated the electronic properties of the single crystals of several tellurides (HgTe and its solid solutions, $\mathrm{HgTe}_{1-x} \mathrm{~S}_{x}, \mathrm{PbTe}$, and $\mathrm{Bi}_{2} \mathrm{Te}_{3}$ ) by the in situ high-pressure measurements up to $\sim 10 \mathrm{GPa}$ under multiple pressure cycling. We found the dramatic changes in their electronic properties in the normalpressure crystalline phases and addressed them to the electronic topological transitions. In particular, we observed the inversions in the electrical conductivity type $(n-p$ or $p-n)$, which were reversible in $\mathrm{HgTe}$, but irreversible in both $\mathrm{PbTe}$ and $\mathrm{Bi}_{2} \mathrm{Te}_{3}$. We proposed simple examples of how these stress-controlled $n-p$ or $p-n$ conductivity switching could be potentially implemented in micro- and nanoelectronic appliances.

\section{ACKNOWLEDGMENTS}

The research was supported by a grant of Ministry of Science and Higher Education of the Russian Federation No. 075-15-2020-797 (13.1902.21.0024). 


\section{DATA AVAILABILITY}

The data that support the findings of this study are available from the corresponding authors upon reasonable request.

\section{REFERENCES}

${ }^{1}$ W. Shi, T. Stedman, and L. M. Woods, J. Appl. Phys. 128, 025104 (2020). ${ }^{2}$ S. V. Ovsyannikov, I. V. Korobeinikov, N. V. Morozova, A. Misiuk, N. V. Abrosimov, and V. V. Shchennikov, Appl. Phys. Lett. 101, 062107 (2012). ${ }^{3}$ S. V. Ovsyannikov, X. Wu, G. Garbarino, M. Núñez-Regueiro, V. V. Shchennikov, J. A. Khmeleva, A. E. Karkin, N. Dubrovinskaia, and L. Dubrovinsky, Phys. Rev. B 88, 184106 (2013).

${ }^{4}$ S. V. Ovsyannikov, A. E. Karkin, N. V. Morozova, V. V. Shchennikov, E. Bykova, A. M. Abakumov, A. A. Tsirlin, K. V. Glazyrin, and L. Dubrovinsky, Adv. Mater. 26, 8185 (2014)

${ }^{5}$ I. V. Korobeinikov, N. V. Morozova, V. V. Shchennikov, and S. V. Ovsyannikov, Sci. Rep. 7, 44220 (2017).

${ }^{6}$ N. V. Morozova, I. V. Korobeinikov, K. V. Kurochka, A. N. Titov, and S. V. Ovsyannikov, J. Phys. Chem. C 122, 14362 (2018).

${ }^{7}$ N. V. Morozova, I. V. Korobeinikov, N. V. Abrosimov, and S. V. Ovsyannikov, CrystEngComm 22, 5416 (2020).

${ }^{8}$ A. T. Duong, V. Q. Nguyen, G. Duvjir, V. T. Duong, S. Kwon, J. Y. Song, J. K. Lee, J. E. Lee, S. Park, T. Min, J. Lee, J. Kim, and S. Cho, Nat. Commun. 7, 13713 (2016).

${ }^{9}$ M. Akasaka, T. Iida, A. Matsumoto, K. Yamanaka, Y. Takanashi, T. Imai, and N. Hamada, J. Appl. Phys. 104, 013703 (2008).

${ }^{10}$ A. Masarrat, A. Bhogra, R. Meena, M. Bala, R. Singh, V. Barwal, C.-L. Dong, C.-L. Chen, T. Som, A. Kumar, A. Niazi, and K. Asokan, RSC Adv. 9, 36113 (2019).

${ }^{11}$ M. Sinduja, S. Amirthapandian, A. Masarrat, R. Krishnan, S. K. Srivastava, and A. Kandasami, Thin Solid Films 697, 137834 (2020).

${ }^{12}$ M. Baleva and E. Mateeva, Phys. Rev. B 50, 8893 (1994).

${ }^{13} \mathrm{M}$. Baleva and M. Momtchilova, Phys. Rev. B 50, 15056 (1994).

${ }^{14}$ T. Wen, Y. Wang, N. Li, Q. Zhang, Y. Zhao, W. Yang, Y. Zhao, and H. Mao, J. Am. Chem. Soc. 141, 505 (2019).

${ }^{15}$ T. Nilges, S. Lange, M. Bawohl, J. M. Deckwart, M. Janssen, H.-D. Wiemhöfer, R. Decourt, B. Chevalier, J. Vannahme, H. Eckert, and R. Weihrich, Nat. Mater. 8, 101 (2009).

${ }^{16}$ O. Osters, M. Bawohl, J.-L. Bobet, B. Chevalier, R. Decourt, and T. Nilges, Solid State Sci. 13, 944 (2011).

${ }^{17}$ C. Xiao, X. Qin, J. Zhang, R. An, J. Xu, K. Li, B. Cao, J. Yang, B. Ye, and Y. Xie, J. Am. Chem. Soc. 134, 18460 (2012).

${ }^{18}$ S. N. Guin, J. Pan, A. Bhowmik, D. Sanyal, U. V. Waghmare, and K. Biswas, J. Am. Chem. Soc. 136, 12712 (2014).

${ }^{19}$ S. N. Guin and K. Biswas, Phys. Chem. Chem. Phys. 17, 10316 (2015).

${ }^{20}$ P. Roy and T. Maiti, J. Phys. D Appl. Phys. 51, 065104 (2018).

${ }^{21}$ O. de la Peña, A. Aguayo, and R. de Coss, Phys. Rev. B 66, 012511 (2002).

${ }^{22}$ J. Zhang, C. Liu, X. Zhang, F. Ke, Y. Han, G. Peng, Y. Ma, and C. Gao, Appl. Phys. Lett. 103, 052102 (2013).

${ }^{23}$ Y. Zhang, Y. Li, Y. Ma, Y. Li, G. Li, X. Shao, H. Wang, T. Cui, X. Wang, and P. Zhu, Sci. Rep. 5, 14681 (2015)

${ }^{24}$ N.-N. Wang, H.-Y. Lu, Z.-T. Lv, W. Wang, J.-Q. He, L. Geng, and S. Chen, J. Low Temp. Phys. 181, 242 (2015).

${ }^{25}$ Z. J. Xiang, G. J. Ye, C. Shang, B. Lei, N. Z. Wang, K. S. Yang, D. Y. Liu, F. B. Meng, X. G. Luo, L. J. Zou, Z. Sun, Y. Zhang, and X. H. Chen, Phys. Rev. Lett. 115, 186403 (2015).

${ }^{26}$ Y. A. Sorb, V. Rajaji, P. S. Malavi, U. Subbarao, P. Halappa, S. C. Peter, S. Karmakar, and C. Narayana, J. Phys. Condens. Matter 28, 015602 (2016).

${ }^{27}$ Y. Qi, W. Shi, P. G. Naumov, N. Kumar, R. Sankar, W. Schnelle, C. Shekhar, F.-C. Chou, C. Felser, B. Yan, and S. A. Medvedev, Adv. Mater. 29, 1605965 (2017).
${ }^{28}$ A. Ohmura, Y. Higuchi, T. Ochiai, M. Kanou, S. Nakano, F. Ishikawa, A. Nakayama, Y. Yamada, and T. Sasagawa, J. Phys. Conf. Ser. 950, 042036 (2017).

${ }^{29}$ T. Nishimura, H. Sakai, H. Mori, K. Akiba, H. Usui, M. Ochi, K. Kuroki, A. Miyake, M. Tokunaga, Y. Uwatoko, K. Katayama, H. Murakawa, and N. Hanasaki, Phys. Rev. Lett. 122, 226601 (2019).

${ }^{30}$ L.-C. Chen, P.-Q. Chen, W.-J. Li, Q. Zhang, V. V. Struzhkin, A. F. Goncharov, Z. Ren, and X.-J. Chen, Nat. Mater. 18, 1321 (2019).

${ }^{31}$ T. Ideue, M. Hirayama, H. Taiko, T. Takahashi, M. Murase, T. Miyake, S. Murakami, T. Sasagawa, and Y. Iwasa, Proc. Natl. Acad. Sci. U.S.A. 116, 25530 (2019).

${ }^{32}$ O. De la Peña-Seaman, R. de Coss, R. Heid, and K.-P. Bohnen, Phys. Rev. B 79, 134523 (2009).

${ }^{33}$ Y. L. Chen, J. G. Analytis, J.-H. Chu, Z. K. Liu, S.-K. Mo, X. L. Qi, H. J. Zhang, D. H. Lu, X. Dai, Z. Fang, S. C. Zhang, I. R. Fisher, Z. Hussain, and Z.-X. Shen, Science 325, 178 (2009).

${ }^{34}$ H. Zhang, C.-X. Liu, X.-L. Qi, X. Dai, Z. Fang, and S.-C. Zhang, Nat. Phys. 5, 438 (2009)

${ }^{35}$ S.-Y. Xu, C. Liu, N. Alidoust, M. Neupane, D. Qian, I. Belopolski, J. D. Denlinger, Y. J. Wang, H. Lin, L. A. Wray, G. Landolt, B. Slomski, J. H. Dil, A. Marcinkova, E. Morosan, Q. Gibson, R. Sankar, F. C. Chou, R. J. Cava, A. Bansil, and M. Z. Hasan, Nat. Commun. 3, 1192 (2012).

${ }^{36}$ L. Wu, M. Brahlek, R. Valdés Aguilar, A. V. Stier, C. M. Morris, Y. Lubashevsky, L. S. Bilbro, N. Bansal, S. Oh, and N. P. Armitage, Nat. Phys. 9, 410 (2013)

${ }^{37}$ W.-J. Shi, J. Liu, Y. Xu, S.-J. Xiong, J. Wu, and W. Duan, Phys. Rev. B 92, 205118 (2015).

${ }^{38}$ J. Liu, Y. Xu, J. Wu, B.-L. Gu, S. B. Zhang, and W. Duan, Acta Crystallogr. C Struct. Chem. 70, 118 (2014).

${ }^{39}$ J. Zhu, A. R. Oganov, W. X. Feng, Y. G. Yao, S. J. Zhang, X. H. Yu, J. L. Zhu, R. C. Yu, C. Q. Jin, X. Dai, Z. Fang, and Y. S. Zhao, AIP Adv. 6, 085003 (2016).

${ }^{40}$ V. Rajaji, P. S. Malavi, S. S. R. K. C. Yamijala, Y. A. Sorb, U. Dutta, S. N. Guin, B. Joseph, S. K. Pati, S. Karmakar, K. Biswas, and C. Narayana, Appl. Phys. Lett. 109, 171903 (2016).

${ }^{41}$ M. Zhang, X. Wang, A. Rahman, Q. Zeng, D. Huang, R. Dai, Z. Wang, and Z. Zhang, Appl. Phys. Lett. 112, 041907 (2018).

${ }^{42}$ N. M. Alsaleh, E. Shoko, M. Arsalan, and U. Schwingenschlögl, Phys. Status Solidi RRL. 12, 1800083 (2018).

${ }^{43} \mathrm{M}$. Hajji, H. Absike, H. Labrim, H. Ez-Zahraouy, M. Benaissa, and A. Benyoussef, Comput. Condens. Matter 16, e00299 (2018).

${ }^{44}$ I. M. Lifshitz, Sov. Phys. JETP 11, 1130 (1960).

${ }^{45}$ A. Polian, M. Gauthier, S. M. Souza, D. M. Trichês, J. Cardoso de Lima, and T. A. Grandi, Phys. Rev. B 83, 113106 (2011).

${ }^{46}$ P. Aguado-Puente, S. Fahy, and M. Grüning, Phys. Rev. Res. 2, 043105 (2020).

${ }^{47}$ S. V. Ovsyannikov, V. V. Shchennikov, A. Y. Manakov, A. Y. Likhacheva, Y. S. Ponosov, V. E. Mogilenskikh, A. P. Vokhmyanin, A. I. Ancharov, and E. P. Skipetrov, Phys. Status Solidi 246, 615 (2009).

${ }^{48} \mathrm{~L}$. Xu, Y. Zheng, and J.-C. Zheng, Phys. Rev. B 82, 195102 (2010).

${ }^{49} \mathrm{~K}$. Akiba, Electronic States of Narrow-Gap Semiconductors Under Multi-Extreme Conditions (Springer Singapore, Singapore, 2019).

${ }^{50}$ A. A. Andreev, J. Phys. Colloq. 29, C4-50-C4-61 (1968).

${ }^{51}$ Y. W. Tung and M. L. Cohen, Phys. Lett. A 29, 236 (1969).

${ }^{52}$ Y. I. Ravich, B. A. Efimova, and I. A. Smirnov, Semiconducting Lead Chalcogenides (Springer US, Boston, MA, 1970).

${ }^{53}$ M. Schlüter, G. Martinez, and M. L. Cohen, Phys. Rev. B 12, 650 (1975).

${ }^{54}$ G. Nimtz and B. Schlicht, Narrow-Gap Semiconductors (Springer, Berlin, 1983), pp. 1-117.

${ }^{55}$ V. V. Shchennikov and S. V. Ovsyannikov, Solid State Commun. 126, 373 (2003).

${ }^{56}$ G. Rousse, S. Klotz, A. M. Saitta, J. Rodriguez-Carvajal, M. I. McMahon, B. Couzinet, and M. Mezouar, Phys. Rev. B 71, 224116 (2005).

${ }^{57}$ V. V. Shchennikov, S. V. Ovsyannikov, A. Y. Manakov, A. Y. Likhacheva, A. I. Ancharov, I. F. Berger, and M. A. Sheromov, J. Exp. Theor. Phys. Lett. 83, 228 (2006). 
${ }^{58}$ Y. Li, C. Lin, H. Li, X. Li, and J. Liu, High Pressure Res. 33, 713 (2013).

${ }^{59}$ Y.-C. Li, G. Li, C.-L. Lin, X.-D. Li, and J. Liu, Chin. Phys. Lett. 32, 016101 (2015).

${ }^{60}$ V. V. Shchennikov, S. V. Ovsyannikov, and A. Y. Derevskov, Phys. Solid State 44, 1845 (2002).

${ }^{61}$ Y. Wang, X. Chen, T. Cui, Y. Niu, Y. Wang, M. Wang, Y. Ma, and G. Zou, Phys. Rev. B 76, 155127 (2007).

${ }^{62}$ S. V. Ovsyannikov and V. V. Shchennikov, Appl. Phys. Lett. 90, 122103 (2007).

${ }^{63}$ A. Svane, N. E. Christensen, M. Cardona, A. N. Chantis, M. van Schilfgaarde, and T. Kotani, Phys. Rev. B 81, 245120 (2010).

${ }^{64}$ P. Barone, T. Rauch, D. Di Sante, J. Henk, I. Mertig, and S. Picozzi, Phys. Rev. B 88, 045207 (2013).

${ }^{65}$ V. V. Shchennikov, S. V. Ovsyannikov, and A. V. Bazhenov, J. Phys. Chem. Solids 69, 2315 (2008).

${ }^{66}$ V. V. Shchennikov, S. V. Ovsyannikov, and A. Y. Manakov, J. Phys. Chem. Solids 71, 1168 (2010).

${ }^{67}$ V. V. Shchennikov, S. V. Ovsyannikov, A. Y. Derevskov, and V. V. Shchennikov, J. Phys. Chem. Solids 67, 2203 (2006).

${ }^{68} \mathrm{~N}$. V. Morozova, V. V. Shchennikov, and S. V. Ovsyannikov, J. Appl. Phys. 118, 225901 (2015).

${ }^{69}$ R. Piotrzkowski, S. Porowski, Z. Dziuba, J. Ginter, W. Giriat, and L. Sosnowski, Phys. Status Solidi B 8, K135 (1965).

${ }^{70}$ I. M. Tsidilkovski, Electron Spectrum of Gapless Semiconductors (Springer Verlag, Berlin, 1996).

${ }^{71}$ N. Orlowski, J. Augustin, Z. Gołacki, C. Janowitz, and R. Manzke, Phys. Rev. B 61, R5058 (2000).

${ }^{72}$ L. Fu and C. L. Kane, Phys. Rev. B 76, 045302 (2007).

${ }^{73}$ A. Svane, N. E. Christensen, M. Cardona, A. N. Chantis, M. van Schilfgaarde, and T. Kotani, Phys. Rev. B 84, 205205 (2011).

${ }^{74}$ M. V. Yakunin, A. V. Suslov, M. R. Popov, E. G. Novik, S. A. Dvoretsky, and N. N. Mikhailov, Phys. Rev. B 93, 085308 (2016).

${ }^{75}$ G. M. Minkov, O. E. Rut, A. A. Sherstobitov, S. A. Dvoretski, N. N. Mikhailov, V. A. Solov'ev, M. Y. Chernov, S. V. Ivanov, and A. V. Germanenko, Phys. Rev. B 101, 245303 (2020).

${ }^{76}$ P. W. Bridgman, Proc. Am. Acad. Arts Sci. 74, 21 (1940).

${ }^{77}$ A. N. Mariano and E. P. Warekois, Science 142, 672 (1963).

${ }^{78}$ A. Jayaraman, W. Klement, and G. C. Kennedy, Phys. Rev. 130, 2277 (1963).

${ }^{79}$ S. Katsuki and M. Kunimune, J. Phys. Soc. Jpn. 31, 337 (1971).

${ }^{80}$ S. Narita, M. Egawa, K. Suizu, M. Katayama, and S. Mizukami, Appl. Phys. 2, 151 (1973).

${ }^{81}$ T. M. Turusbekov and E. I. Estrin, Sov. Phys. Solid State 21, 558 (1979).

${ }^{82}$ A. Ohtani, T. Seike, M. Motobayashi, and A. Onodera, J. Phys. Chem. Solids 43, 627 (1982).

${ }^{83}$ A. Werner, H. D. Hochheimer, K. Strössner, and A. Jayaraman, Phys. Rev. B 28, 3330 (1983).

${ }^{84}$ T. Huang and A. L. Ruoff, Phys. Status Solidi 77, K193 (1983).

${ }^{85}$ S. B. Qadri, A. W. Webb, E. F. Skelton, N. Moulton, J. Furdyna, and L. Colombo, High Pressure Res. 4, 303 (1990).

${ }^{86} \mathrm{~N}$. G. Wright, M. I. McMahon, R. J. Nelmes, and A. San-Miguel, Phys. Rev. B 48, 13111 (1993).

${ }^{87}$ A. San-Miguel, N. G. Wright, M. I. McMahon, and R. J. Nelmes, Phys. Rev. B 51, 8731 (1995)

${ }^{88}$ R. J. Nelmes and M. I. McMahon, Semicond. Semimetals 54, 145 (1998).

${ }^{89}$ Y. S. Ponosov, V. V. Shchennikov, V. E. Mogilenskikh, V. I. Osotov, and S. V. Popova, Phys. Status Solidi 223, 275 (2001).

${ }^{90}$ D. P. Kozlenko, V. P. Glazkov, S. Hull, B. N. Savenko, V. V. Shchennikov, and V. I. Voronin, Appl. Phys. A Mater. Sci. Process. 74, s983 (2002).

${ }^{91}$ D. P. Kozlenko, V. V. Shchennikov, V. I. Voronin, V. P. Glazkov, and B. N. Savenko, Phys. Solid State 44, 1628 (2002).

${ }^{92}$ M. Cardona, R. K. Kremer, R. Lauck, G. Siegle, A. Muñoz, and A. H. Romero, Phys. Rev. B 80, 195204 (2009).

${ }^{93}$ S. Radescu, A. Mujica, J. López-Solano, and R. J. Needs, Phys. Rev. B 83, 094107 (2011).
${ }^{94}$ X. D. Hu, D. H. Zhu, Z. F. Zeng, and S. R. Sun, Adv. Mater. Res. 1004-1005, 1608 (2014).

${ }^{95}$ T.-L. Huang and A. L. Ruoff, Phys. Rev. B 31, 5976 (1985).

${ }^{96}$ X. Chen, Y. Wang, T. Cui, Y. Ma, G. Zou, and T. Iitaka, J. Chem. Phys. 128, 194713 (2008).

${ }^{97}$ T. Ouyang and M. Hu, J. Appl. Phys. 117, 245101 (2015).

${ }^{98}$ J. Blair and A. C. Smith, Phys. Rev. Lett. 7, 124 (1961).

${ }^{99}$ I. M. Tsidil'kovskii, V. V. Shchennikov, and N. G. Gluzman, Sov. Phys. Solid State 24, 1507 (1982).

${ }^{100}$ V. V. Shchennikov and N. G. Gluzman, Sov. Phys. Solid State 23, 1800 (1981).

${ }^{101}$ I. M. Tsidil'kovskii, V. V. Shchennikov, and N. G. Gluzman, Sov. Phys. Semicond. 17, 604 (1983).

${ }^{102}$ S. W. McKnight and M. K. El-Rayess, Solid State Commun. 54, 433 (1985).

${ }^{103}$ V. V. Shchennikov, N. P. Gavaleshko, and V. M. Frasunyak, Phys. Solid State 35, 199 (1993).

${ }^{104} \mathrm{~V}$. V. Shchennikov, Phys. Solid State 35, 401 (1993).

${ }^{105}$ F. M. Shchennikov, V. V. Gavaleshko, and N. P. Frasunyak, Phys. Solid State 37, 1943 (1995).

${ }^{106}$ T.-J. Hu, X.-Y. Cui, X.-F. Li, J.-S. Wang, X.-M. Lv, L.-S. Wang, J.-H. Yang, and C.-X. Gao, Chin. Phys. B 24, 116401 (2015).

${ }^{107}$ V. V. Shchennikov, A. E. Kar'kin, N. P. Gavaleshko, and V. M. Frasunyak, Phys. Solid State 42, 215 (2000).

${ }^{108}$ B. Al Shafaay, F. El Haj Hassan, and M. Korek, Comput. Mater. Sci. 83, 107 (2014).

${ }^{109}$ T. Rauch, S. Achilles, J. Henk, and I. Mertig, Phys. Rev. Lett. 114, 236805 (2015).

${ }^{110}$ X. Dai, T. L. Hughes, X.-L. Qi, Z. Fang, and S.-C. Zhang, Phys. Rev. B 77, 125319 (2008)

${ }^{111}$ F. Kirtschig, J. van den Brink, and C. Ortix, Phys. Rev. B 94, 235437 (2016).

${ }^{112}$ A. Laref, M. Alsagri, Z. Hussain, S. Laref, F. Tawfik, and S. J. Luo, J. Mater. Sci. 52, 7039 (2017).

${ }^{113}$ V. V. Shchennikov, S. V. Ovsyannikov, and N. Y. Frolova, J. Phys. D Appl. Phys. 36, 2021 (2003).

${ }^{114}$ O. Falkenbach, M. O. Loeh, C. W. Wiegand, A. Schmitz, D. Hartung, G. Koch, P. J. Klar, E. Mueller, and S. Schlecht, J. Electron. Mater. 46, 5781 (2017).

${ }^{115}$ H. Öztürk, G. G. Arslan, C. Kürkçü, and Ç Yamçıçıer, J. Electron. Mater. 49, 3089 (2020).

${ }^{116}$ F. Demiray and S. Berber, Phys. Scr. 88, 015603 (2013).

${ }^{117}$ P. W. Bridgman, Proc. Am. Acad. Arts Sci. 76, 55 (1948).

118 A. N. Mariano and K. L. Chopra, Appl. Phys. Lett. 10, 282 (1967).

${ }^{119}$ I. Wakabayashi, H. Kobayashi, H. Nagasaki, and S. Minomura, J. Phys. Soc. Jpn. 25, 227 (1968).

${ }^{120}$ Y. Fujii, K. Kitamura, A. Onodera, and Y. Yamada, Solid State Commun. 49, 135 (1984).

${ }^{121} \mathrm{~T}$. Chattopadhyay, H. G. von Schnering, W. A. Grosshans, and W. B. Holzapfel, Physica B+C 139-140, 356 (1986).

${ }^{122}$ S. Ves, Y. A. Pusep, K. Syassen, and M. Cardona, Solid State Commun. 70, 257 (1989).

${ }^{123}$ M. K. Jacobsen, R. S. Kumar, and A. L. Cornelius, J. Electron. Mater. 41, 633 (2012).

${ }^{124}$ M. K. Jacobsen, W. Liu, and B. Li, J. Phys. Condens. Matter 25, 365402 (2013).

${ }^{125}$ J. Baker, R. Kumar, C. Park, C. Kenney-Benson, A. Cornelius, and N. Velisavljevic, J. Synchrotron Radiat. 23, 1368 (2016)

${ }^{126}$ S. Ma, C. Guo, C. Xiao, F. Wu, M. Smidman, Y. Lu, H. Yuan, and H. Wu, Adv. Funct. Mater. 28, 1803188 (2018).

${ }^{127}$ S. V. Ovsyannikov and V. V. Shchennikov, Chem. Mater. 22, 635 (2010).

${ }^{128}$ T. H. Hsieh, H. Lin, J. Liu, W. Duan, A. Bansil, and L. Fu, Nat. Commun. 3, 982 (2012).

${ }^{129}$ D. Bassanezi, E. O. Wrasse, and T. M. Schmidt, Mater. Res. Express 5, 015051 (2018)

${ }^{130}$ E. P. Skipetrov, E. A. Zvereva, O. S. Volkova, A. V. Golubev, A. Y. Mollaev, R. K. Arslanov, and V. E. Slyn'ko, Semiconductors 38, 1164 (2004). 
${ }^{131}$ N. B. Brandt, D. V. Gitsu, N. S. Popovich, V. I. Sidorov, and S. M. Chudinov, JETP Lett. 22, 104 (1975).

${ }^{132}$ D. A. Wright, Nature 181, 834 (1958).

${ }^{133} \mathrm{~N}$. Serebryanaya, E. Tatyanin, S. Buga, I. Kruglov, N. Lvova, and V. Blank, Phys. Status Solidi 252, 267 (2015).

${ }^{134}$ S. G. Buga, V. A. Kulbachinskii, V. G. Kytin, G. A. Kytin, I. A. Kruglov, N. A. Lvova, N. S. Perov, N. R. Serebryanaya, S. A. Tarelkin, and V. D. Blank, Chem. Phys. Lett. 631-632, 97 (2015).

${ }^{135}$ X. Guo, X. Jia, K. Jie, H. Sun, Y. Zhang, B. Sun, and H. Ma, Chem. Phys. Lett. 568-569, 190 (2013).

${ }^{136}$ A. N. Veis, L. N. Luk'yanova, and V. A. Kutasov, Phys. Solid State 54, 2182 (2012).

${ }^{137}$ V. G. Orlov, G. S. Sergeev, and E. A. Kravchenko, J. Magn. Magn. Mater. 475, 627 (2019)

${ }^{138}$ K. Kusagaya, H. Hagino, S. Tanaka, K. Miyazaki, and M. Takashiri, J. Electron. Mater. 44, 1632 (2015).

${ }^{139}$ M. Takashiri, K. Kurita, H. Hagino, S. Tanaka, and K. Miyazaki, J. Appl. Phys. 118, 065301 (2015).

${ }^{140}$ V. R. Akshay, B. Arun, M. V. Suneesh, and M. Vasundhara, ACS Appl. Nano Mater. 1, 3236 (2018)

${ }^{141}$ P. Fourmont, L. F. Gerlein, F.-X. Fortier, S. G. Cloutier, and R. Nechache, ACS Appl. Mater. Interfaces 10, 10194 (2018).

${ }^{142}$ J. Shi, H. Chen, S. Jia, and W. Wang, Mater. Lett. 212, 299 (2018).

${ }^{143}$ A. Gaul, Q. Peng, D. J. Singh, T. Borca-Tasciuc, and G. Ramanath, J. Appl. Phys. 125, 165101 (2019).

${ }^{144}$ G. Rogl, M. J. Zehetbauer, and P. F. Rogl, Mater. Trans. 60, 2071 (2019).

${ }^{145}$ S. Bano, A. Kumar, B. Govind, A. H. Khan, A. Ashok, and D. K. Misra, J. Mater. Sci. Mater. Electron. 31, 8607 (2020).

${ }^{146}$ J. L. Zhang, S. J. Zhang, H. M. Weng, W. Zhang, L. X. Yang, Q. Q. Liu, S. M. Feng, X. C. Wang, R. C. Yu, L. Z. Cao, L. Wang, W. G. Yang, H. Z. Liu, W. Y. Zhao, S. C. Zhang, X. Dai, Z. Fang, and C. Q. Jin, Proc. Natl. Acad. Sci. U.S.A. 108, 24 (2011)

${ }^{147}$ C. Zhang, L. Sun, Z. Chen, X. Zhou, Q. Wu, W. Yi, J. Guo, X. Dong, and Z. Zhao, Phys. Rev. B 83, 140504 (2011).

${ }^{148}$ S. J. Zhang, J. L. Zhang, X. H. Yu, J. Zhu, P. P. Kong, S. M. Feng, Q. Q. Liu, L. X. Yang, X. C. Wang, L. Z. Cao, W. G. Yang, L. Wang, H. K. Mao, Y. S. Zhao, H. Z. Liu, X. Dai, Z. Fang, S. C. Zhang, and C. Q. Jin, J. Appl. Phys. 111, 112630 (2012).

${ }^{149}$ S. V. Ovsyannikov, V. V. Shchennikov, G. V. Vorontsov, A. Y. Manakov, A. Y. Likhacheva, and V. A. Kulbachinskii, J. Appl. Phys. 104, 053713 (2008).

${ }^{150}$ S. V. Ovsyannikov, N. V. Morozova, I. V. Korobeinikov, L. N. Lukyanova, A. Y. Manakov, A. Y. Likhacheva, A. I. Ancharov, A. P. Vokhmyanin, I. F. Berger, O. A. Usov, V. A. Kutasov, V. A. Kulbachinskii, T. Okada, and V. V. Shchennikov, Appl. Phys. Lett. 106, 143901 (2015).

${ }^{151}$ A. Nakayama, M. Einaga, Y. Tanabe, S. Nakano, F. Ishikawa, and Y. Yamada, High Pressure Res. 29, 245 (2009).

${ }^{152}$ M. Einaga, A. Ohmura, A. Nakayama, F. Ishikawa, Y. Yamada, and S. Nakano, Phys. Rev. B 83, 092102 (2011).

${ }^{153}$ W. Ibarra-Hernández, M. J. Verstraete, and J.-Y. Raty, Phys. Rev. B 90, 245204 (2014).

${ }^{154}$ M. K. Jacobsen, S. V. Sinogeikin, R. S. Kumar, and A. L. Cornelius, J. Phys. Chem. Solids 73, 1154 (2012).

${ }^{155} \mathrm{~K}$. Zhao, Y. Wang, C. Xin, Y. Sui, X. Wang, Y. Wang, Z. Liu, and B. Li, J. Alloys Compd. 661, 428 (2016).
${ }^{156}$ F. J. Manjón, R. Vilaplana, O. Gomis, E. Pérez-González, D. Santamaría-Pérez, V. Marín-Borrás, A. Segura, J. González, P. Rodríguez-Hernández, A. Muñoz, C. Drasar, V. Kucek, and V. Muñoz-Sanjosé, Phys. Status Solidi 250, 669 (2013).

${ }^{157}$ R. Vilaplana, O. Gomis, F. J. Manjón, A. Segura, E. Pérez-González, P. Rodríguez-Hernández, A. Muñoz, J. González, V. Marín-Borrás, V. Muñoz-Sanjosé, C. Drasar, and V. Kucek, Phys. Rev. B 84, 104112 (2011).

${ }^{158}$ G. K. Pradhan, A. Bera, P. Kumar, D. V. S. Muthu, and A. K. Sood, Solid State Commun. 152, 284 (2012).

${ }^{159}$ H. Zhu, J. Dong, P. Li, Y. Wang, Z. Guo, X. Shan, Y. Gong, P. An, X. Li, J. Zhang, and D. Chen, Phys. Status Solidi 254, 1700007 (2017).

${ }^{160}$ E. Y. Atabaeva, E. S. Itskevich, S. A. Mashkov, S. P. Popova, and L. F. Vereshchagin, Sov. Phys. Solid State USSR 10, 43 (1968).

${ }^{161}$ L. F. Vereshchagin, N. A. Bendeliani, and E. Y. Atabaeva, Sov. Phys. Solid State USSR 13, 2051 (1972).

${ }^{162}$ L. G. Khvostantsev, A. I. Orlov, N. Kh. Abrikosov, T. E. Svechnikova, and S. N. Chizhevskaya, Phys. Status Solidi 71, 49 (1982).

${ }^{163}$ L. Zhu, H. Wang, Y. Wang, J. Lv, Y. Ma, Q. Cui, Y. Ma, and G. Zou, Phys. Rev. Lett. 106, 145501 (2011)

${ }^{164}$ G. Xiao, K. Wang, L. Zhu, X. Tan, Y. Qiao, K. Yang, Y. Ma, B. Liu, W. Zheng, and B. Zou, J. Phys. Chem. C 119, 3843 (2015).

${ }^{165}$ J. Zhao, Z. Yu, Q. Hu, Y. Wang, J. Schneeloch, C. Li, R. Zhong, Y. Wang, Z. Liu, and G. Gu, Phys. Chem. Chem. Phys. 19, 2207 (2017).

${ }^{166}$ A. Gaul, Q. Peng, D. J. Singh, G. Ramanath, and T. Borca-Tasciuc, Phys. Chem. Chem. Phys. 19, 12784 (2017).

${ }^{167}$ V. V. Brazhkin and A. I. Orlov, JETP Lett. 99, 283 (2014).

${ }^{168}$ C.-Y. Moon and S.-H. Wei, Phys. Rev. B 74, 045205 (2006).

${ }^{169}$ R. Sakuma, C. Friedrich, T. Miyake, S. Blügel, and F. Aryasetiawan, Phys. Rev. B 84, 085144 (2011).

${ }^{170}$ S. H. Groves, R. N. Brown, and C. R. Pidgeon, Phys. Rev. 161, 779 (1967).

${ }^{171}$ K. Seeger, Semiconductor Physics (Springer, New York, 1973).

${ }^{172}$ Y. Pei, X. Shi, A. LaLonde, H. Wang, L. Chen, and G. J. Snyder, Nature 473, 66 (2011).

${ }^{173}$ A. V. Dmitriev, Mater. Res. Express 6, 015512 (2018).

${ }^{174}$ B. M. Goltsman, B. A. Kudinov, and I. A. Smirnov, Thermoelectric Semiconductor Materials Based on Bi2Te3 (Nauka, Moscow, 1972).

${ }^{175}$ H. Köhler, Phys. Status Solidi 74, 591-600 (1976).

${ }^{176}$ V. A. Kulbachinskii, N. B. Brandt, P. A. Cheremnykh, S. A. Azou, J. Horak, and P. Lošták, Phys. Status Solidi 150, 237 (1988).

${ }^{177}$ V. A. Kulbachinskii, N. E. Klokova, J. Gorak, P. Lostjak, S. A. Azou, and G. A. Mironova, Fiz. Tverd. Tela 31, 205 (1989).

${ }^{178}$ S. K. Mishra, S. Satpathy, and O. Jepsen, J. Phys. Condens. Matter 9, 461 (1997).

${ }^{179}$ V. A. Greanya, W. C. Tonjes, R. Liu, C. G. Olson, D.-Y. Chung, and M. G. Kanatzidis, Phys. Rev. B 62, 16425 (2000).

${ }^{180}$ A. Kadir, T. Ganguli, R. Kumar, M. R. Gokhale, A. P. Shah, S. Ghosh, B. M. Arora, and A. Bhattacharya, Appl. Phys. Lett. 91, 111913 (2007).

${ }^{181}$ S. Ruffell, K. Sears, J. E. Bradby, and J. S. Williams, Appl. Phys. Lett. 98, 052105 (2011).

${ }^{182}$ S. Reboh, R. Coquand, S. Barraud, N. Loubet, N. Bernier, G. Audoit, J.-L. Rouviere, E. Augendre, J. Li, J. Gaudiello, N. Gambacorti, T. Yamashita, and O. Faynot, Appl. Phys. Lett. 112, 051901 (2018). 